Kürzere Beiträge

\title{
Albrecht von Haller im Urteil Alexander von Humboldts*
}

\author{
Von Markus Breuning
}

1.

Albrecht von Haller lebte 1708-1777, Alexander von Humboldt 1769-1859; persönlich sind sich die beiden universalen Geister nie begegnet. Aber schon der junge Humboldt lernte Werke Hallers kennen. Das Sommersemester 1789 verbrachte er als Student in Göttingen, wo Haller 1736-1753 als Professor gewirkt hatte. Dort hörte Humboldt die Physikvorlesungen Georg Christoph Lichtenbergs, der im Alter zwischen beiden stand (1742-1799). Aus Hamburg, wo Humboldt an der Handelsakademie weiterstudierte, schrieb er am 22. Februar 1791 einen Brief an Lichtenberg über seine Gedanken zur Salzkochung mit weniger Holzverbrauch ${ }^{1}$. Die Frage hatte ihn in einer Vorlesung Lichtenbergs gepackt. Mit derselben Aufgabe hatte sich Haller befaßt, als er 1758-1764 Salzdirektor in Roche war, und er hatte nach eigenen Versuchen die Sonnenverdunstung empfohlen.

Haller verarbeitete sein «Mémoire sur l'évaporation de l'eau salée» (Histoire de l'Académie Royale des Sciences, Paris 1764) deutsch in seiner «Beschreibung der Salzwerke in dem Amte Aelen» (Bern 1765). Diese wurde in ein Buch «Bemerkungen über Schweizerische Salzwerke» aufgenommen und mit vielen Zusätzen erweitert herausgegeben von Karl Christian Langsdorf (Leipzig 1789) ${ }^{2}$. Diese Ausgabe benützte Humboldt. Die Brüder Johann Wilhelm Langsdorf (1745 bis 1827) und Karl Christian Langsdorf (1757-1834) waren in Humboldts Jugendzeit in der Salzwerkskunde führend ${ }^{3}$.

Nach dem Abschluß seiner Studien an der Bergakademie in Freiberg trat Humboldt 1792 in den preußischen Staatsdienst als Assessor beim Berg- und Hüttendepartement ein. Minister Friedrich Anton von Heinitz (1725-1802), der Chef des preußischen Bergwesens, wollte Humboldt «zu einer maßgebenden Instanz für das Salinenwesen heranbilden.$^{4}$ Auf einer amtlichen Reise sollte er Erfahrungen über Salzgradierung und Salzfindung sammeln.

Im «Versuch über einige chemische und physikalische Grundsätze der Salzwerkkunde $»^{5}$ nannte Humboldt Haller fünfmal. Auf Seite 33 schrieb er : «Hr. von Haller nahm unbedingt an, daß durch das Gradiren auf Dornwänden 1/3 der

\footnotetext{
* Herrn Jürg Aufenast in Dankbarkeit gewidmet.

Von Heinz Balmer gekürzte Fassung.
} 
Soole verloren gehe... Herr Langsdorf hat diese verwickelten Verhältnisse neuerlichst durch eine sinnreiche, mit der Erfahrung übereinstimmende Formel dargestellt. (S. Hallers Bemerk. 1789. S. 102. wo der Calcül in der Sammlung praktischer Bemerk. für Freunde der Salzwerkskunde, Th. 2, S. 218. und in der Anleit. zur Salzwerkskunde, 1784. S. 61 berichtigt wird.)»

Verwiesen ist hier auf Johann Wilhelm und Karl Christian Langsdorfs «Sammlung practischer Bemerkungen und einzelner zerstreuter Abhandlungen für Freunde der Salzwerkskunde» (1785) und Karl Christian Langsdorfs «Vollständige auf Theorie und Erfahrung gegründete Anleitung zur Salzwerkskunde» (5 Bände, Altenburg 1784-1796).

Eine weitere Stelle findet sich auf Seite 37: «Der kostspielige Bau der Lekwerke, der zunehmende Mangel an Dornen, die Ersparung sämmtlicher Bewegkräfte und der mannichfaltige Soolenverlust beym Gradiren, brachten Herrn von Haller ums Jahr 1759 auf die Idee, die zu Nauheim (1579) erfundenen ... Gradirhäuser zu verlassen. Er versuchte statt derselben die Sonnengradirung, wie in Poitou und Pays d'Aunis, doch in bedachten hölzernen und marmornen Bassins, einzuführen ... (S. Langsdorfs Anmerk. zu Haller. S. 168.) Herr von Haller berechnete aus diesen und ähnlichen Erfahrungen die Vortheile der Sonnengradirung, und schloß, daß dieselbe die Gradirung auf Dornwänden sechsmal an Vortheil übertreffe. Aber Hr. Langsdorf ... folgert ..., daß nur in Gegenden, wo die Soole sparsam, Feurung, Dornen und Bewegkräfte aber überaus kostbar sind, der hallersche Plan ein vorzügliches Augenmerk verdiene.»

Auf Hallers Bemerkungen über Schweizerische Salzwerke 1789 verweist Humboldt ebenso auf den Seiten 2, 24 und 26 seiner Abhandlung.

\section{2.}

Das zweite Gebiet, auf dem Humboldt sich mit Haller traf, war die Pflanzenphysiologie. Seine «Aphorismen aus der chemischen Physiologie der Pflanzen» erschienen zuerst lateinisch als Anhang zum «Florae Fribergensis specimen» (Berlin 1793, S. 133-182) und dann 1794 in Leipzig in der deutschen Übersetzung von Gotthelf Fischer. Das Büchlein möchte die Pflanzenkunde von der bloßen Systematik erlösen und zur Pflanzenphysiologie erweitern. So ist es nicht verwunderlich, daß Humboldt den damals immer noch gebräuchlichen Physiologieleitfaden Hallers zu Rate zog. Haller hatte ihn 1747 lateinisch als «Primae lineae physiologiae» für seine Göttinger Studenten herausgegeben, und die Auflagen waren sich bis in sein Alter gefolgt. 1780 besorgte der Göttinger Anatomieprofessor Heinrich August Wrisberg eine mit Anmerkungen erweiterte Ausgabe, die die letzte lateinische blieb. Die erste deutsche Übersetzung, an der Haller sich noch 
selber beteiligt hatte, war 1770 in Berlin erschienen; die weiteren von 1781 an trugen den Titel «Grundriß der Physiologie» und schlossen Wrisbergs Zusätze ein. Humboldt benützte die lateinische Ausgabe von 1780 , aber auch die damals neueste deutsche Ausgabe von 1788, die nicht nur mit Wrisbergs Zusätzen versehen, sondern überdies von dem Mainzer Anatomieprofessor Samuel Thomas Sömmerring erläutert war. Außerdem kannte Humboldt Hallers Hauptwerk, die «Elementa physiologiae corporis humani» (8 Bände, Lausanne und Bern 1757 bis 1766). Er benützte auch ihre deutsche Übersetzung von Johann Samuel Halle, «Anfangsgründe der Physiologie des menschlichen Körpers» (8 Bände, Berlin 1759-1776), sowie Hallers zweite lateinische Ausgabe «De partium corporis humani praecipuarum fabrica et functionibus» (8 Bände, Bern 1777-1778) ${ }^{6}$.

An der ersten Hauptstelle (S. 24-26) bemerkt Humboldt, die alternde Muskelfiber komme dem Holze sehr nahe. Aus zwei Zitaten, die er aus den «Primae lineae» übersetzte, erfahren wir Hallers Ansicht, daß die alternden Muskeln sich in harte, nicht mehr reizbare Sehnen verwandeln. Humboldt fügt bei, daß andere Anatomen (Andreas Murray, Sömmerring, Johann Friedrich Meckel und John Hunter) dieser Meinung widersprochen hätten. Die Muskelfasern würden zwar im alternden Menschen dichter und zäher, nähmen jedoch die Natur der Sehnen nicht an. Trotzdem läßt Humboldt gelten: «Was dieser große Mann uns von alternden Fibern sagt, das geschieht auch, wie uns die tägliche Erfahrung lehrt, in den Pflanzen. Die belebten Gefäße verengen sich, gehen von Bast und Splint in Holz über, werden dichter, und nehmen, wenn die Mischung der Grundstoffe nach den Gesetzen der chemischen Verwandtschaft vor sich gegangen ist, die Natur unbelebter Theile an.»

Haller betrachtete als Grundbaustein der Pflanzen und Tiere die Fiber. Humboldt bemerkt (S. 31), dagegen habe Abilgaard in der Hydra keine Fibern, sondern nur Bläschen wahrgenommen.

Die zweite Hauptstelle findet sich auf den Seiten 43-45. «Angenehm ist mir die Betrachtung, daß schon der unsterbliche Haller denjenigen beitrat, welche den Vegetabilien Reizbarkeit zuschreiben.» Dies wird mit einem längeren, aus den «Primae lineae» übersetzten Zitate belegt. Es schließt mit Hallers Worten über jene Kraft: «Sie hat ihren Sitz blos in der weichen Fiber. Mit der Verhärtung derselben hingegen verschwindet sie.»

\section{3.}

Am nächsten kam Humboldt Haller, als er sich auf das Feld des physiologischen Experimentes begab. Da er jedoch die elektrische Reizung untersuchte, also just jenes Verfahren übte, das erst nach Hallers Tode von Luigi Galvani 1780 ent- 
deckt und 1791 und 1792 bekanntgegeben worden war, mußten ihm Hallers Ausführungen überholt und die jüngsten Fortschritte der Wissenschaft groß erscheinen. Der 28jährige Humboldt veröffentlichte seine Ergebnisse in den zwei Bänden «Versuche über die gereizte Muskel- und Nervenfaser nebst Vermuthungen über den chemischen Process des Lebens in der Thier- und Pflanzenwelt» (Posen/Berlin 1797 und 1798).

Im Frühling 1793 hatte Humboldt seinen Dienst als Oberbergmeister in Bayreuth angetreten; kurz nach seiner Beförderung zum Oberbergrat im Mai 1795 schrieb er am 7.Juni aus Bayreuth an Sömmerring: «Ich war indeß meist mit Seciren von Thieren, mit Versuchen über Gifte und Metallreiz beschäftigt. Ich habe Ihre Schriften und die große Physiologie von Haller fleißig studirt und glaube, manches neue entdekt zu haben. ${ }^{7}$

«Voll Optimismus bekennt er sich zur Fortschrittlichkeit», bestätigt Heinrich Schipperges ${ }^{8}$. Humboldt vergleicht in seinem Buch von 1797 das jüngsterworbene Wissen mit dem zu Hallers Zeit: «Wer den Fortschritten der Physiologie belebter Thier- und Pflanzenstoffe gefolgt ist, den darf ich laut auffordern, das, was der große Haller über die sogenannten Lebensgeister gesammelt hat [Große Physiologie, Bd. 4, S. 563-618], mit dem zu vergleichen, was wir am Schlusse dieses Jahrhunderts davon wissen. Wer konnte vormals nur die Möglichkeit fassen, daß die unmittelbare Berührung eines Muskels mit seinem inserirten Nerven eine Muskular-Contraction erregen, oder daß man aus den Organen viele hundert und tausend Fuß weit die thierische Flüssigkeit ableiten kann, von welcher die fibröse Bewegung abhängt ? ${ }^{9}$

Von Hallers «Grundriß der Physiologie» benützte Humboldt ständig die Ausgabe von Sömmerring (1788), wie er Seite 17 angibt. Dort wird eine Stelle über die Ursache der Röte des Blutes zitiert. Auf Seite 188 wird die Zahl erwähnt, die Haller als Geschwindigkeit des Nervensaftes vorstellte ${ }^{10}$, und auf Seite 222 ist vom Nervengeflecht in der Haut die Rede, wozu Band 4 der «Anfangsgründe der Physiologie» herangezogen wird.

Haller hatte die Reizbarkeit der Muskeln und die Empfindlichkeit der Nerven als zwei verschiedene Grundkräfte bezeichnet. Nerven sollten zur Reizbarkeit überflüssig sein. «Wenn man Hallers Schriften nachlieset, so findet man, daß der große Mann, der die Natur, mit welcher er so innigst vertraut war, nie nach seinen Lieblingsideen ummodelte, sich dieser Beweisart nur immer mit Bescheidenheit und Schüchternheit bediente. Seine spätern Nachfolger aber drückten sich entscheidender darüber aus. » (S. 251f.)

In dieser Beziehung ist Haller auch auf den Seiten 236 und 273 genannt. Dann wird auf Seite 278 Hallers Behauptung erörtert, daß man an keinem Tiere Augen 
ohne Gehirn und Gehirn ohne Augen wahrgenommen habe (Physiologie, Bd. 4, S. 2). «Ich muß dem großen Manne seit meiner Zergliederung der Naiden widersprechen. Die Nais proboscidea zeigt keinen deutlichern Nervenknoten im Kopfe, als die Nais vermicularis, und diese ist blind, während daß jene zwei schöne schwarze Augen besitzt.» Naiden sind durchsichtige Borstenwürmer, die an Wasserpflanzen leben.

Auch auf Seite 339 setzt sich Humboldt kritisch mit Haller auseinander. Dieser habe seine Lehre von der eigentümlichen, von der Nervenfaser unabhängigen Muskelreizbarkeit vor allem auch auf die wurmförmige Bewegung des Darmes gestützt. Aber: «Warum will man über den schönen Bau der vier Darmhäute und ihre Faserschichten, das ansehnliche Netz der bis in die Zellstoffhaut zu verfolgenden ... zahlreichen Nerven aus dem Auge verlieren? ... Unausführbar ist es daher, wie es Haller fordert, den Darmkanal, remotis nervis zu reizen; jedes mechanische Stechen in die Muskelschicht eines Eingeweides trift wahrscheinlich einen Nerven selbst, oder seinen sensiblen Wirkungskreis.»

Auf Seite 63 des zweiten Bandes bestreitet Humboldt die Ansicht Hallers, daß die Reize auf das Gluten wirkten, da die Muskelfaser aus Erde und Gluten zusammengesetzt sei. «In einem belebten Organe ist alles belebt. Die vitalen Functionen hängen daher nicht von einzelnen Stoffen, sondern von der Zusammenwirkung aller ab. Wir dürfen uns nicht einzelne Substanzen auswählen und diesen zuschreiben, was das gemeinsame Resultat der ganzen Mischung ist. » Einig war er dagegen mit Hallers Ansicht, daß die Gefäße auf die Reizbarkeit der Muskelfaser Einfluß hätten. Er schreibt auf Seite 68 des zweiten Bandes: «Die Gefäße, die sich (wie schon der unsterbliche Haller lehrte) so unendlich wichtig für die Stimmung der Irritabilität zeigen, sind, je nachdem sie andere und andere Säfte hervorbringen, schon allein im Stande, die Mischung der Muskelfaser zu modificiren.»

Ein Hinweis auf Hallers Opera minora, Tomus III, S. 191, folgt auf Seite 99. Weitere Erwähnungen Hallers bieten die Seiten 379 und 412.

\section{4.}

Mit seiner großen Reise nach Amerika (1799-1804) und ihrer Auswertung entfernte sich Humboldt von Hallers Arbeitsgebiet. Nur noch selten nennt er ihn. In einem Fach aber stand Humboldt auch als Geograph unter der Fortwirkung Hallers, nämlich in der Pflanzengeographie. Heinrich Zoller hat bemerkt: «Besonders wirksam waren Hallers botanische Leistungen in pflanzengeographischer Hinsicht. Im Vorwort zur 'Historia stirpium' (1768) gibt er auf einer einzigen Seite seine überaus glückliche, geniale Synthese zwischen den Höhenstufen der 
Alpen und den gürtelförmigen Zonen der Vegetation und Flora Eurasiens ... Die lebendige Schilderung war nicht nur von größtem Einfluß auf H. B. de Saussure, sondern hat später im 19. Jahrhundert auch Alexander von Humboldt entscheidend angeregt. ${ }^{11}$ Eine Stelle, die nur in der französischen Ausgabe des «Essai sur la géographie des plantes» (Paris 1805; Bd. XXVII des amerikanischen Reisewerkes, S. 74) vorkommt, betrifft das Moosglöcklein und lautet: «Le Linnaea borealis, qui, près de Berlin, en Suède, aux Etats-Unis et à Nootka-Sund, se trouve au niveau de la mer, croît dans les Alpes de la Suisse à cinq cents et sept cents mètres (256 et 359 toises) d'élévation. On le découvre au Valais, au bord du torrent qui coule sous la Tête-Noire; au S. Gothard, où Haller l'a observé le premier. ${ }^{12}$

Humboldts Reisebericht erschien 1814, 1819 und 1825 in drei großen Bänden unter dem Titel «Relation historique du Voyage aux Régions équinoctiales du Nouveau Continent». Der Name Hallers findet sich nur an zwei Stellen des ersten Bandes. Am Anfang des fünften Kapitels sagt Humboldt über das spanische Amerika, die dortigen Halbgelehrten hätten mit einer gewissen Geringschätzung auf ihn und Bonpland herabgesehen, wenn sie hörten, daß sich unter ihren Büchern das «Spectacle de la nature» von Abbé Pluche, der «Cours de physique» von Sigaud La Fond und der «Dictionnaire» von Valmont de Bomare nicht befanden. Diese drei Werke und der «Traité d'économie politique» von Jakob Friedrich Bielfeld seien die bekanntesten und geachtetsten fremden Bücher im Gebiet von Caracas und Chile bis Guatemala und Nordmexiko; «et c'est seulement dans les grandes capitales, à Lima, à Santa-Fe de Bogota, et à Mexico, que les noms de Haller, de Cavendish et de Lavoisier commencent à remplacer ceux dont la célébrité est devenue populaire depuis un demi-siècle ». ${ }^{13}$

Die zweite Erwähnung steht in einer Anmerkung der französischen Ausgabe ${ }^{14}$. Im Dorfe Arenas bei Cumana hörte Humboldt von einem Bauern Francisco Lozano, der seinen Sohn mit seiner eigenen Milch aufgezogen habe. Da die Mutter erkrankt war, drückte der Vater das Kind, um es zu beruhigen, an seine Brust. Infolge der Reizung der Brustwarze, an der das Kind saugte, begann die Brust Milch abzusondern. Der Vater säugte das Kind fünf Monate lang. Seine Brust wurde in Cumana von Bonpland untersucht. «Il n'est pas très-rare de trouver, parmi les hommes et les animaux, des mâles dont les mamelles renferment du lait», fügt Humboldt bei und verweist auf Hallers Elementa, Band VII, Pars II, S. 18.

In seinem Alterswerk, dem «Kosmos», hat Humboldt im Kapitel über die Entwicklung der Naturschilderung auch Haller einen Platz angewiesen. Er lobt ihn als Dichter nicht überschwänglich, gibt ihm aber gegenüber seinen dichtenden 
Zeitgenossen den Vorrang. Die Stelle ${ }^{15}$ lautet: «In unserm deutschen Vaterlande hat sich das Naturgefühl wie in der italiänischen und spanischen Litteratur nur zu lange in der Kunstform des Idylls, des Schäferromans und des Lehrgedichts offenbart. Auf diesem Wege wandelten oft der persische Reisende Paul Flemming, Brockes, der gefühlvolle Ewald von Kleist, Hagedorn, Salomon Geßner und einer der größten Naturforscher aller Zeiten, Haller, dessen locale Schilderungen wenigstens bestimmtere Umrisse und eine mehr objective Wahrheit des Colorits darbieten.» Durch die ehrenvolle Beifügung vor seinem Namen hat Haller in Humboldts Kosmos ein würdiges Denkmal erhalten.

\section{Anmerkungen}

1 Briefe Alexander von Humboldts aus seiner Frühzeit an Georg Christoph Lichtenberg. In: Sudhoffs Archiv 32 (1939) 399-408; hier S. 401 f.

2 Susanna Lundsgaard-Hansen-von Fischer, Verzeichnis der gedruckten Schriften Albrecht von Hallers, Bern 1959, S. 49 f.

${ }^{3}$ Siehe ihre Lebensabrisse in der Allgemeinen Deutschen Biographie, Bd. 17.

${ }^{4}$ Hanno Beck, Alexander von Humboldt, 2 Bde., Wiesbaden 1959 und 1961; hier Bd. I, S. 51.

5 Erschienen in: Alexander Wilhelm Köhler und Christian August Siegfried Hoffmann, Bergmännisches Journal, Freyberg und Annaberg, Jg. 5, 1792, Bd. I, Stück 1, S. 1-45, und Stück 2, S. 97-141. - Die Titel der Arbeiten Humboldts sind zusammengestellt bei: Julius Löwenberg, Alexander von Humboldt. Bibliographische Übersicht seiner Werke, Schriften und zerstreuten Abhandlungen. In: Karl Bruhns (Hrsg.), Alexander von Humboldt. Eine wissenschaftliche Biographie, 3 Bde., Leipzig 1872; Bd. 2, S. 485-552; auch separat als Neudruck, Verlag F.A.Brockhaus, Stuttgart 1960, 68 S.

6 In den "Aphorismen» zitiert Humboldt S. 8, 25, 26, 31 und 44 die «Primae lineae» von 1780, S. 26 den "Grundriß» von 1788, S. 26 und 31 den Band 4 der "Anfangsgründe», S. 31 und 41 den Band 4 der «Elementa » und S. 52 den Band 8 der «Fabrica».

7 Beiträge zur Alexander von Humboldt-Forschung 2/1973, Berlin 1973: Die Jugendbriefe A.v. Humboldts 1787-1799 (Bearbeiter: Ilse Jahn, Fritz Lange), Brief 303, S. 428.

${ }^{8}$ Heinrich Schipperges, Alexander von Humboldt und die Medizin seiner Zeit. In: Archiv für Kulturgeschichte 41 (1959) 166-182; hier S. 172.

9 Versuche über die gereizte Muskel- und Nervenfaser I, S. 455 f.

10 Dazu auch: Heinrich Schipperges, Quellen zu Humboldts medizinischem Weltbild. In: Sudhoffs Archiv 43 (1959) 147-171; hier S. 162.

11 Heinrich Zoller, Albrecht von Haller als Botaniker. In: Aktuelle Probleme aus der Geschichte der Medizin (Verhandlungen des XIX. Internationalen Kongresses für Geschichte der Medizin, Basel 1964), Basel/New York 1966, S. 461-463; hier S. 463.

12 Hinweis von Fritz G. Lange.

13 Relation historique, S. 320 f. (Neudruck in: Quellen und Forschungen zur Geschichte der Geographie und der Reisen, hrsg. von Hanno Beck, Verlag F.A.Brockhaus, Stuttgart 
1970). Deutsche Übersetzung von Hermann Hauff: Reise in die Aequinoctial-Gegenden des neuen Continents, 4 Bde., Stuttgart 1859-1860; hier Bd. 1, S. 247.

14 Hinweis von Hanno Beck. Die Stelle findet sich in der Relation historique, S. 376, und in der deutschen Übersetzung von Hermann Hauff, Bd. 1, S. $310 \mathrm{f}$.

${ }^{15}$ Kosmos. Entwurf einer physischen Weltbeschreibung. Bd. 2, Stuttgart und Tübingen 1847, S. 68.

\section{Summary}

Humboldt studied many of Haller's works. Though he paid respect to "the immortal Haller", he criticized some of his opinions. But he appreciated that Haller never tried to model nature according to his personal ideas (Muskel- und Nervenfaser I, p. 251).

Markus Breuning

Liebeggweg 15

3006 Bern 